\title{
THE ACTIVITY OF MALIGNANT TUMOURS AND RESPONSE TO THERAPEUTIC AGENTS, STUDIED BY CONTINUOUS RECORDS OF RADIOACTIVE PHOSPHORUS UPTAKE
}

\author{
M. A. Bullen, B.Sc. H. F. Freundlich, M.A. Brendan T. Hale, M.B., Ch.B., D.M.R.T. \\ D. H. Marshall, Ph.D. \\ R. C. TudwaY, B.Sc., M.B., B.S., F.F.R.
}

United Bristol Hospitals

For some time past, there has been a need for a method of selecting hormone-dependent breast cancers because of the increasing use of hypophysectomy, as well as prolonged therapy by hormones. Without selection at least $50 \%$ of the cases would derive no benefit from such surgery, but up till now one has had to be content with a partial selection based on response to trials of hormone therapy or oophorectomy. The work to be described sprang from our attempt to meet that need.

Our first introduction, in this Department, to the use of radioactive-phosphorus uptake into the growth as a guide to activity, arose in two cases of secondary breast carcinoma in the eyeball. The uptake was assessed by placing a Geiger counter on the eyeball after administering a tracer dose of ${ }^{32} \mathrm{P}$. It was found that activity diminished after the administration of hormones and deep X-ray therapy.

This simple method of surface counting is, however, useless for deep tumours, and unsatisfactory for many superficial ones, because of the short range of the beta rays, the serious errors due to small changes in position of the counters and the effects of changes of surface temperature and blood flow. The method has only been used when it is essential, e.g. in some early melanomata or very superficial skin nodules. Whenever possible, the use of a counter buried in the depth of a tumour has been preferred. The possibility of following tumour activity, after trial administration of hormones by means of serial biopsy and repeated mitotic counts, was quickly rejected as unsuitable for general clinical use. As well as using the counter buried in the growth, it was felt that continuous recording of the ${ }^{32} \mathrm{P}$ uptake was essential to detect rapid or transient changes.

\section{Biochemical Background}

Alma Howard (1956) has shown by auto- radiography and chromatography that the maximum concentration of ${ }^{32} \mathrm{P}$ in an individual cell occurs in prophase and that this corresponds to the maximum concentration of DNA. However, phosphorus is also largely concerned in cell respiration and the phosphorylation of glucose, and there is some evidence for an interrelationship between glycolysis and nucleic acid synthesis. It may well be, therefore, that the 'peaks' of ${ }^{32} \mathrm{P}$ uptake demonstrated below, while they are possibly associated with mitosis, are mainly due to phosphorus taking part in glycolysis. This view may be supported by the fact that a mechanism for clearance of the phosphorus after the 'peaks' appears to be present to allow of the rapid drop. This is consistent with the fact that the phosphorylation reactions result in the formation of inorganic phosphate, which may be cleared from the cells (Burk, Laszlo, Hunter, Wight and Woods r960). It is known that tumour cells may ferment glucose in great quantities compared with normal ones. This effect will heighten the peaks of ${ }^{32} \mathrm{P}$ uptake.

\section{Physical Aspects \\ Introduction}

A good technique for the instrumental recording of tumour activity should observe a constant tissue volume and be minimally sensitive to changes in the biological site of this sample volume. It must be insensitive to, or capable of distinguishing, environmental changes (e.g. background or circulatory changes) both within and outside the patient, and it must be compatible with long periods, up to several weeks, of observation. Also, of course, it must be well tolerated by the patient.

A detector placed in a uniform concentration of ${ }^{32} \mathrm{P}$ will respond almost solely to radiation originating in a concentric belt of thickness equal to the maximum beta range $(8 \mathrm{~mm}$.). However, most of the counts $(90 \%$ of them) arise 


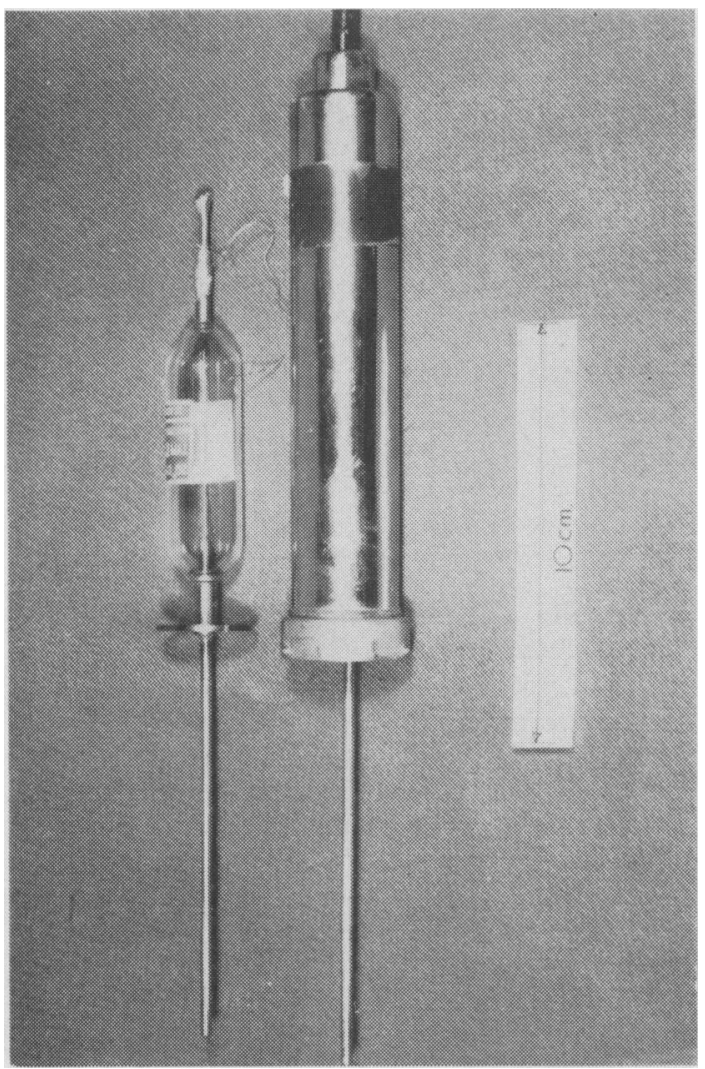

Fig. 1.- ' Needle' G.M. counter for introduction into tumour through the skin.

within $2.5 \mathrm{~mm}$. of the detector. This corresponds to a sample volume of approximately 0.5 c.c. (with our present detectors).

The effective half-life of the ${ }^{32} \mathrm{P}$ in tissues is usually shorter than the physical half-life of 14.2 days, though in a few organs which concentrate phosphorus it may be longer.

\section{Detectors}

The detector is by far the most important component of all. The reliability and complexity of the whole electronic system are governed by the detector. Detectors of the Geiger-Muller, proportional, scintillation and semi-conductor types are applicable, in principle, to our work. At the present moment, however, there are very few which are available in a suitable practical form. We have used needle G.M. counters (Twentieth Century Type NIc) as probes for introduction into the tissues through a skin puncture (Fig. I), and capsule counters (G.M. tube, Mullard MX 150/MX I5I (Fig. 2) for implantation at open operation.

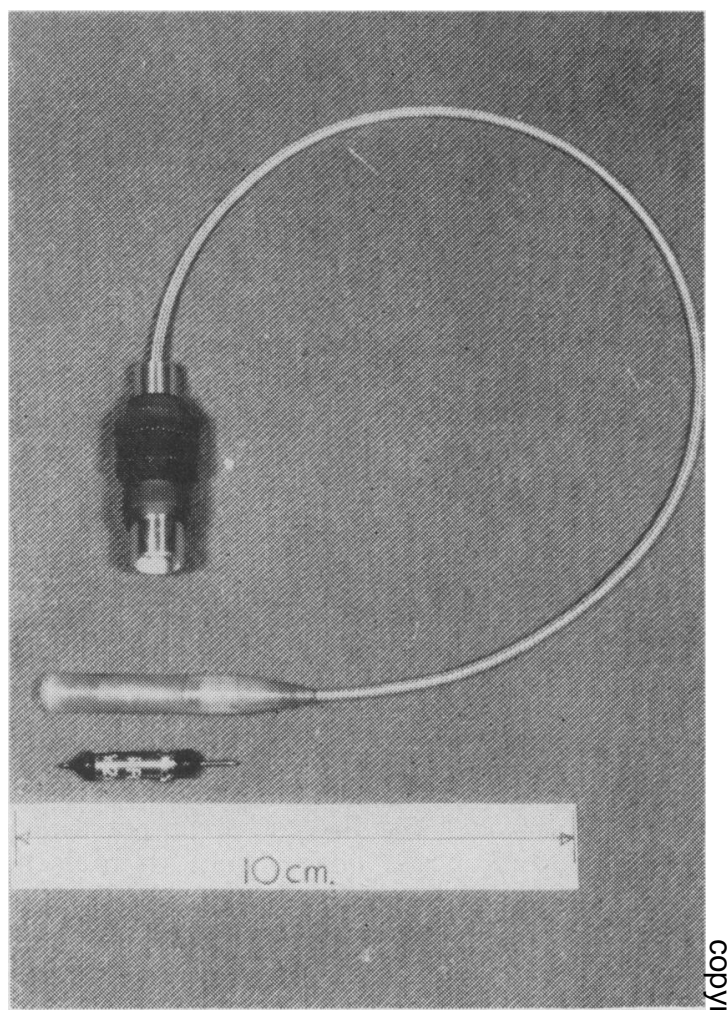

Fig. 2. - ' Capsule' G.M. counter for implantation intoo tumour at operation.

The needle counters have their own disadvantages. The short working-life has been improved by suitable circuit techniques, and in appropriate cases by arranging the switching mechanism so that the counter works intermittently, e.g. by reading for a few minutes every half-hour, or other chosen interval. Fragility has been dealt with by sheathing the whole counter in a stronger cannula, except for the last centimetre of the sensitive end. The counter, in fact, takes the position of a trocar, after insertion of a trocar and cannula.

\section{Bremsstrahlung Counting}

Some tumours remain inaccessible to the easy insertion of a counter, e.g. carcinoma of the bronchus. In these cases the use of ${ }^{32} \mathrm{P}$ uptake studies may still be possible by the use of Bremsstrahlung counting. This may well turn out to be a satisfactory method because the low quantum energy of the rays simplifies the collimation problems associated with external counting. We are working on this. Mehl (1958, 1959) and Kellershohn Herzberg and Martin (1958) have already applied this technique to in-vivo problems. 


\section{Instrumentation}

For the production of the continuous record of uptake, use may be made of either ratemeters or scalers. We have found a scaler more suitable than a ratemeter. The count can be recorded either by a digital printout system or graphically by means of an analogue unit.

We have constructed multichannel recording units because frequently a second control probe is needed in normal tissues, or another part of the tumour, and the ratio of these counts is required. Also a third probe outside the patient (suitably screened to compensate for absence of tissue) will record external background. In addition, we hope to measure temperature, blood flow and oxygen tension in the tumours in some cases.

\section{Statistics}

Radioactive disintegration is a random process, therefore no counting observation can be of absolute accuracy, and each result must be interpreted according to the laws of probability. In our present work the number of counts recorded at each observation ranges between 200 and 5,000 . Thus if one chooses, say, a ' $I$ in 80' probability there are 79 chances in 80 (or nearly a $99 \%$ confidence level) that an observation lies within about plus or minus $18 \%$ or $3 \frac{1}{2} \%$ respectively of the mean value. This appreciation of the probable significance of a reading is essential, in order to decide whether a peak is, or is not, biologically real.

\section{Clinical Programme}

\section{Method of Insertion of the Probes}

The needle portion of the probe is sterilized chemically for the required time and it has been found convenient to use a tall cylindrical measuring glass for this purpose. Consideration is then given to the site of insertion, bearing in mind the problem of fixation of the tube and handle for a considerable period, so as to avoid any unnecessary movement which may decrease the reliability of the counting. If two probes are inserted into the same tumour it is often a good plan to use a short and long reach combination, so as to record from widely separated points, but still have the handles strapped together. A local anæsthetic is used and a stab wound made, followed by a track through which the probe is inserted. It is then held in place by a foam plastic support and waterproof strapping. A control probe may be inserted in a similar way. The technique does necessitate keeping the patient in bed and somewhat immobilized for a time, but does not hinder sleep or produce any troublesome pain (Fig. 3).

The capsule-type counter may be buried in the tissues, an intra-abdominal mass for example and,

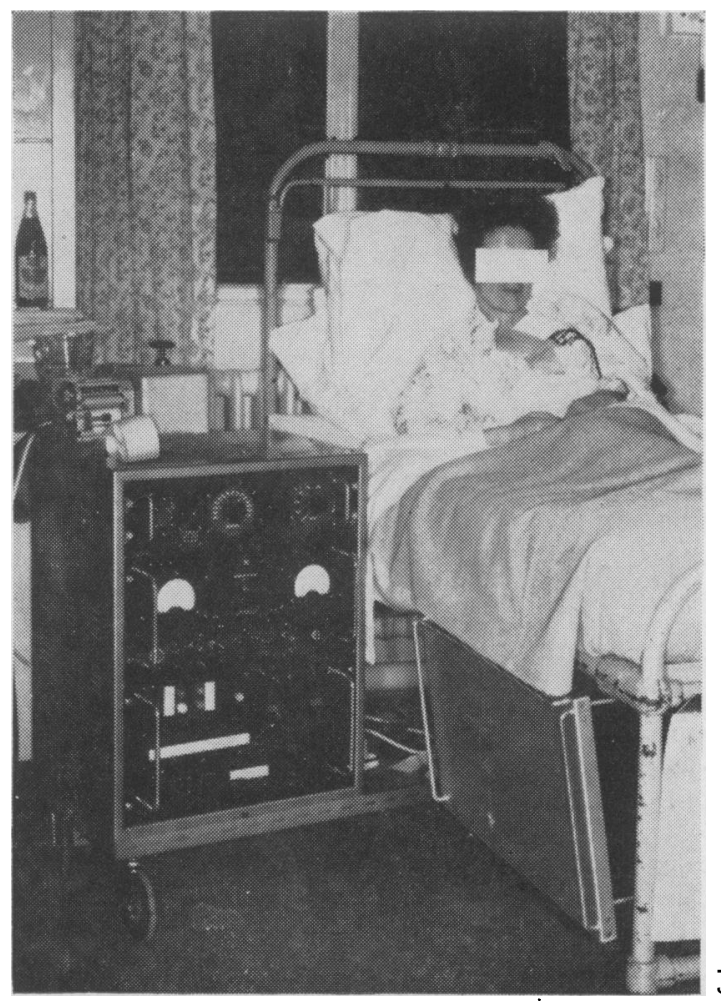

FIG. 3.-'Needle' counter in position in a breast tumour, with older type of recording gear alongside.

after suture of the incision, the waterproof lead protrudes from a small wound. These counter tubes are hermetically sealed into a thin-walled Perspex capsule. The cable has a nylon sheath, so that the whole assembly is non-toxic and may be left in the tissues for prolonged times. The main disadvantage of this counter is the large diameter (7 mm.) although count-life, total bulk and cost are much better than those of the needle counters.

Adsorption effects are a common problem with all implanted detectors particularly where phosphorus is employed. Initially efforts were made to minimize adsorption by a variety of surface coatings. In practice, however, satisfactory working is achieved by inserting counters into the tumour approximately one hour before the ${ }^{32} \mathrm{P}$ dose is given, thus allowing clotting to occur, which isolates the counter to some degree from direct contact with active fluids.

\section{Breast Carcinoma}

Continuous ${ }^{32} \mathrm{P}$ uptake records were first made in breast cancers for the reasons explained above. Also they are accessible for counting and readily available in the Radiotherapy Department. In the 


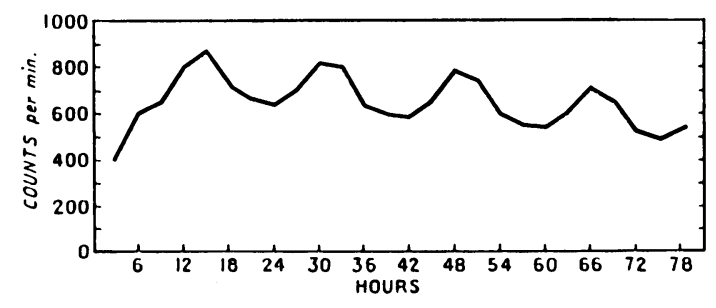

FIG. 4--A typical tracing, showing a regular cycle of growth activity in a hormone-sensitive breast cancer.

first group of cases, records were made from different areas of the same tumour and from the opposite breast, used as a control. In some cases secondary nodules were counted as well as the primary mass. An almost immediate and most striking finding was that in many cases, which were later proved to be hormone-dependent, regular periodic cycles of activity were observed (Fig. 4).

In the first series of 75 breast cases a regular cycle was found in 25 and each of these later proved to be hormone-dependent. No regular cycle was found in 44 cases, but 22 of these also proved to be hormone-dependent.

The remaining six cases showed no regular cycle and very little activity. These were all examples of atrophic scirrhus in old patients with a history of extremely slow growth. The record of these cases was therefore consistent with the clinical type.

The regular cycle, when found, appeared to vary from 6 to 36 hours, with an average frequency of 13 hours. Some, but not all cases, fall in line with diurnal body temperature variations. From these results it appeared that, although cases showing a regular cycle were hormone-dependent, looking for a cycle alone was not a sufficient test, because of the hormone-dependent cases which did not show a cycle of ${ }^{32} \mathrm{P}$ uptake.

The next group of cases demonstrate the effect of the administration of hormones. In some cases this altered the shape of the uptake tracing, and in the majority of these cases hormonedependence was later confirmed (Figs. 5 and 6).

It was found that stimulation of a hormonedependent tumour occurred more quickly than inhibition via the pituitary. In cases where œstrogens produced a favourable response, the depression of activity was often not recorded for at least four days, whereas stimulation usually occurred within 48 hours and in some cases as early as 12 hours after giving the stilbœstrol. Substances used during this test include stilbœstrol, testosterone phenyl proprionate and

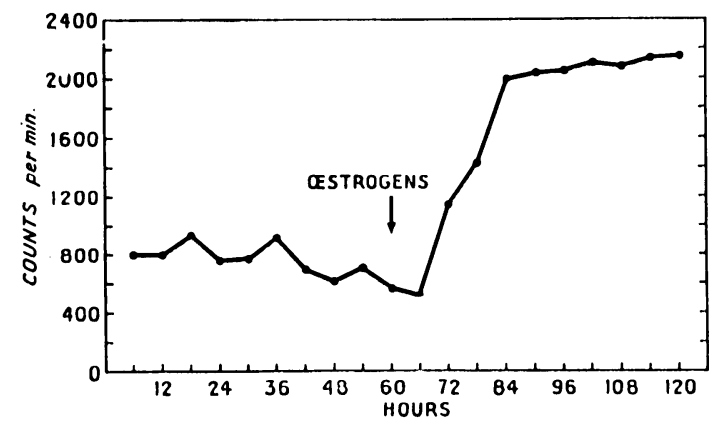

Fig. 5.- The response of an oestrogen-dependent breast tumour to that hormone. The counting-rate has greatly increased within twenty-four hours.

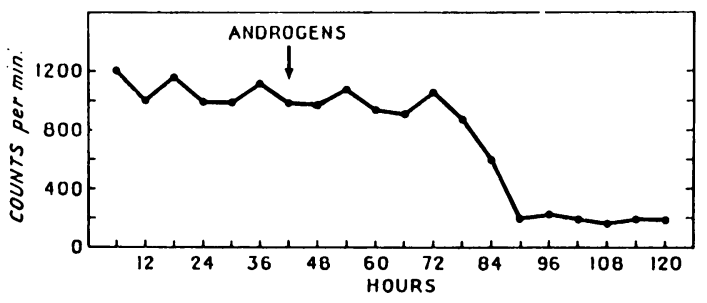

FIG. 6.-The rate of growth of an ostrogen-dependent breast tumour treated with androgens.

pituitary gonadotrophin inhibitors such as norethisterone acetate. At least 48 hours were allowed for recording the base-line before attempting to alter the curve with any drugs. Interpretation of the tracings is not always easy, and the following criteria have been used for forecasting results :

(a) If a regular cycle is seen, hormone-dependence is probable.

(b) If there is no regular cycle, but the mean level of the uptake is altered by hormones, hormone-dependence is also probable.

(c) If there is a regular cycle and also alteration in level of the curve by hormones, hormonedependence is still more likely.

(d) In the absence of any of these findings over a period of at least a week hormonedependence is unlikely.

The following results were obtained when cases were fully assessed for cyclical pattern as well as alteration in activity produced by hormones: 57 cases were fully studied, 52 correct predictions were made; three cases produced equivocal tracings where it was found impossible to make a forecast; and two cases had incorrect forecasts.

Confirmation of results was obtained by either hormone-depriving surgery ( 17 cases) or 


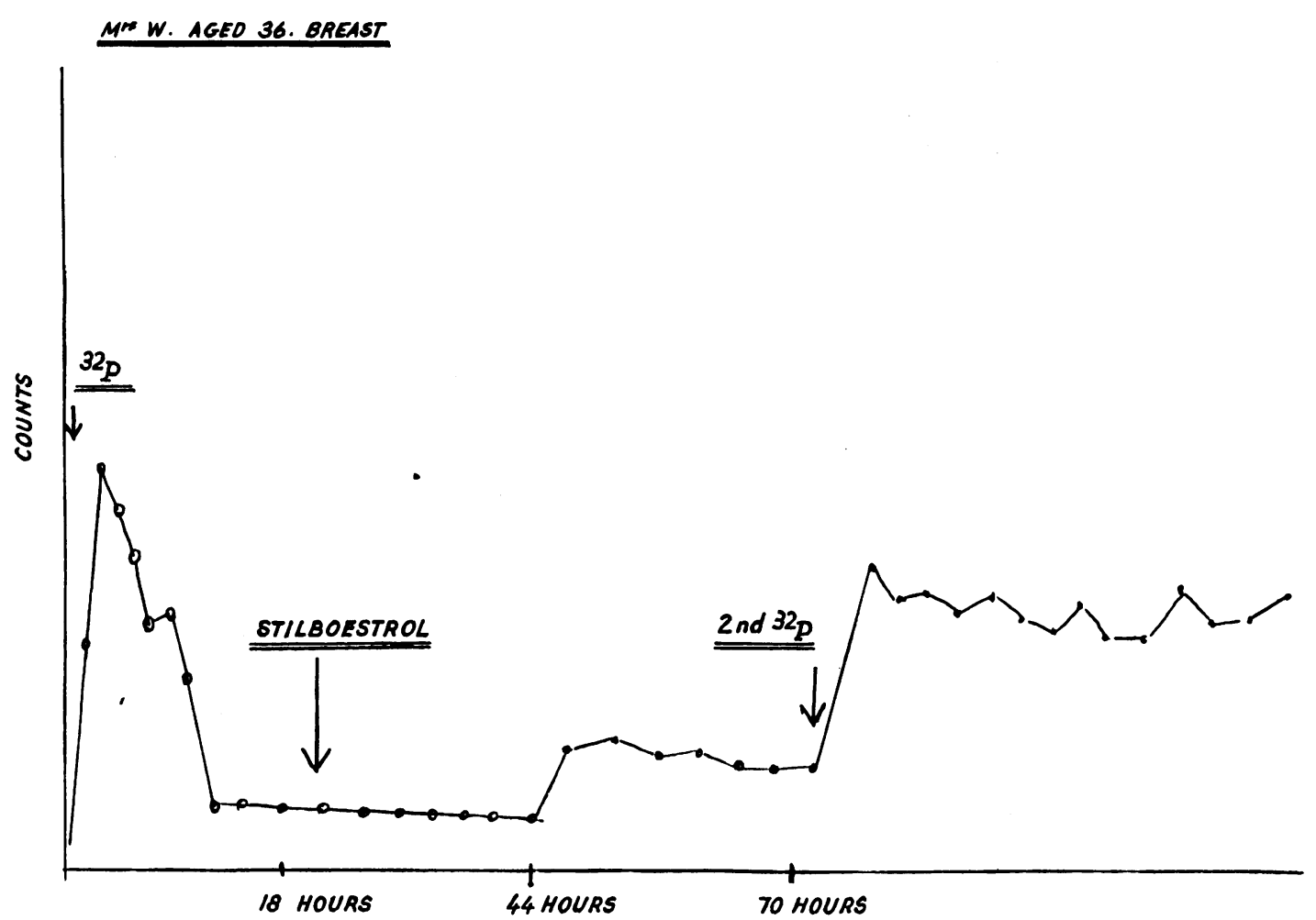

FIG. 7.- Tracing from case of breast carcinoma. Note low count rate following menstrual period (at 18 hours), and rise, with rhythmic peaks of activity after administration of stilbostrol.

by the prolonged use of the appropriate hormones or pituitary gonadotrophic inhibitor. In no case was the forecast allowed to interfere with the planned treatment of the case. In this way it was felt that the results would be more significant and an experimental technique would in no way prejudice the patients' treatment.

An interesting tracing was obtained from a young woman with breast carcinoma. She had noticed that her tumour was more painful in the week prior to menstruation and less painful afterwards. Very little of the first dose of ${ }^{32} \mathrm{P}$ given was held in the tumour, but after administration of stilbœstrol for 24 hours the uptake increased and a second dose of ${ }^{32} \mathrm{P}$ given later was retained in the growth in the usual way (Fig. 7).

\section{Malignant Melanoma}

The next tumours to be studied were malignant melanomas. The object in the first instance was to assess the response of the tumour to chemotherapy. The response of such a case to intravenous Melphalan is shown in Fig. 8. There was a marked fall-off in ${ }^{32} \mathrm{P}$ uptake within 48 hours, though it was four weeks before clinical

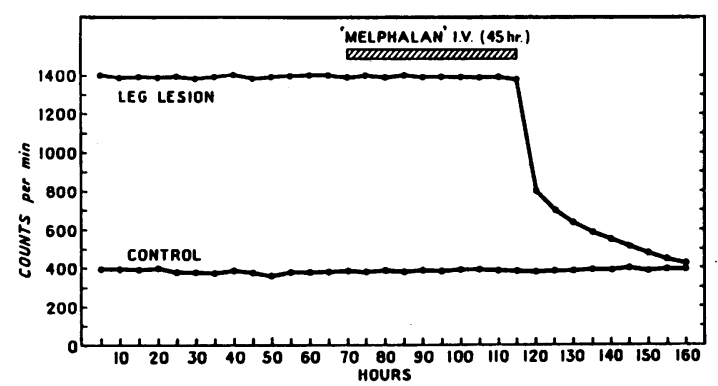

FIG. 8.-The response of a malignant melanoma to chemotherapy.

regression was obvious. An account of the clinical work up to this point has already been published (Hale, 196r).

A surprise finding in other melanomas was that a few of them showed a cyclical tracing. This was similar to the finding in hormonedependent breast cases.

Case No. 1.- In the first of these melanoma cases the administration of $15 \mathrm{mg}$. of stilbœstrol t.d.s. much 


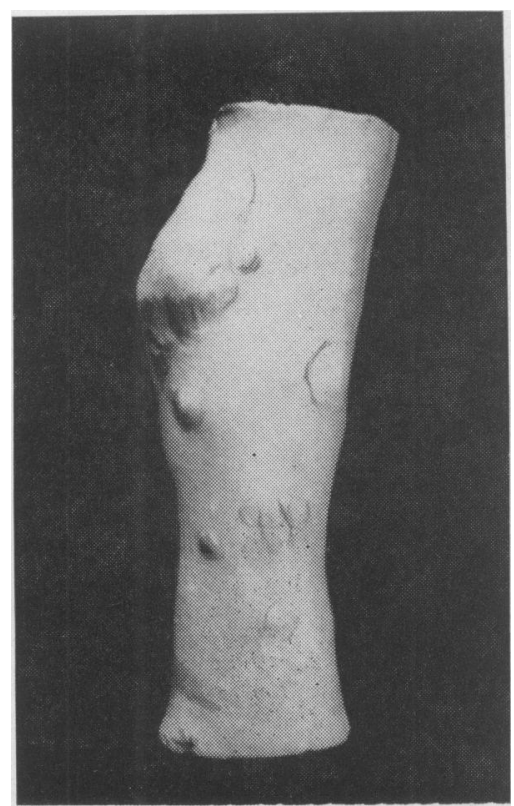

FIG. 9.-Case No. 4 (malignant melanoma): Plaster cast of tumour in leg before treatment.

increased the activity disclosed by a needle-probe placed in one of the many metastatic nodules. The patient was then put on androgens (intramuscular testosterone) and after four months the nodules had disappeared. A change was then made to oral androgens, but after this the nodules recurred along with a cerebral secondary. Further uptake-testing confirmed that the growth was still hormone sensitive. A change back to large doses of testosterone was, however, followed by hæmorrhage into the cerebral secondary, from which the patient died.

Case No. 2.-A second case, which showed a hormone-dependent type of tracing, had a hypophysectomy performed by Mr. Angell James. Within a few weeks her multiple tumours had disappeared and she remained well for nine months. Recurrence then occurred and she died.

Case No. 3.-In a third case the activity of the growth diminished on administration of ostrogens, and extensive melanomatoús nodules regressed on stilbœstrol therapy. She has remained well for ten months on this treatment.

Case No. 4.- In the fourth case hypophysectomy was again carried out. This extraordinary patient was a woman aged 95 . She had a large mass of ulcerated, recurrent metastatic melanoma in the left thigh, as well as smaller deposits. Three weeks after the operation the tumour began to regress. Two months later the ulcer had healed and the masses had gone. A ${ }^{32} \mathrm{P}$ tracing then showed no significant activity at the tumour site. She remained free from recurrence until she died from senility and heart failure seven months later (Figs. 9 and 10 ).

In all, some 26 cases of melanoma have been studied and these four have shown definite evidence of hormone-dependence. The hormonedependence of this tumour has been much

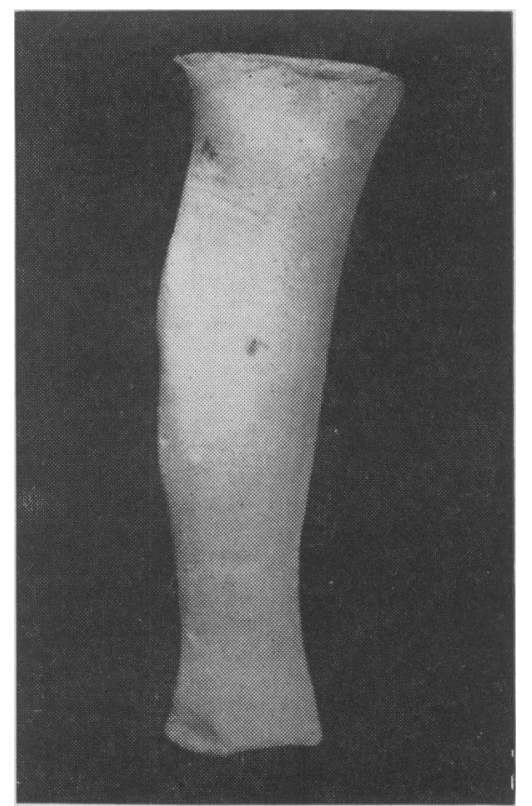

FIg. 10.-Case No. 4: Plaster cast of tumour shown in previous figure, two months after hypophysectomy (hormone-dependent melanoma).

debated and certainly poor results have usuall $\frac{0}{\mathrm{~g}}$ followed hormone-depriving operations. Ouf results suggest that a few melanomas are hormone? dependent. However, our proportion of 4 out of 26 cases is too high, because in some preliminary evidence of hormone sensitivity had been discovered elsewhere.

\section{Radiation Response and the Natural Cycle}

Quite apart from the evidence of hormonedependence which the occurrence of activity peaks provides, another interesting possibility was apparent. Since cells in mitosis are presumed to be more sensitive to radiation than those in the resting stage, and since it was possible that the activity peaks occur at or before the time of waves of mitotic activity in the tumour, it was an obvious idea to try the effect of radiotherapy given only at the time of peaks of ${ }^{32} \mathrm{P}$ uptake. This was done in Case No. 5 (Fig. I I).

The patient was a woman with an advanced carcinoma of the breast which had shown spontaneous activity peaks in the massive growth fixed to the chest wall. There were widespread metastases in the liver and elsewhere. The growth had ulcerated centrally around the nipple area and involved all four quadrants. The breast was divided into two equal halves and a wax jig constructed. Arrangements were made to treat the upper and lower halves to the same dosage, but at different stages of the cycle. The 


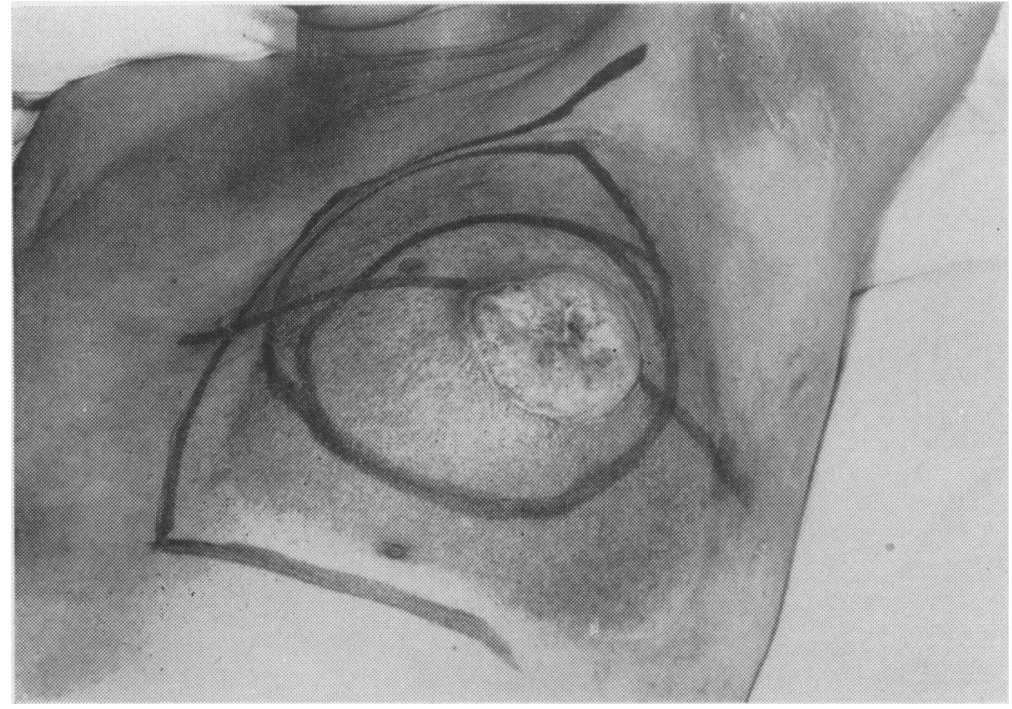

Fig. I I.-Case No. 5: Carcinoma of the breast which had shown natural peaks of ${ }^{32} \mathrm{P}$ uptake, marked to show extent of visible tumour and of palpable tumour (outer ring). The part above the horizontal line was treated with D.X.R. at the time of the rise of each uptake peak. The part below the line was treated between the times of the peaks.

results showed marked regression of the upper half with almost no change in the lower. The upper was treated as the activity indicated by the curve increased, and the lower as it fell. The photograph of the lesion (Fig. 12) shows clearly how much greater response there was in the part of the tumour which was treated at the commencement of the activity peaks. We concluded from this that there was advantage in giving radiotherapy at chosen points in the cycle.

The next problem was to extend the method to other types of growth which do not show natural peaks of activity. As a first step the effect of cooling was investigated in the hope that inhibition of all tumour activity might be followed by a wave of activity by many of the cells when the tumour was allowed to warm-up again, i.e. by the production of an artificial 'peak'.

\section{The Artificial Production of Rhythmic Activity and Response to Radiation}

Case No. 6.-A patient was chosen who had disseminated lymphosarcoma, including a $12-\mathrm{cm}$. diameter mass in the right thigh. Both Geiger probes and a thermocouple were buried in the tumour and a bizarre pattern of activity was seen. The leg was then cooled with surface ice bags and a temperature of $24^{\circ} \mathrm{C}$. was maintained at a depth of $2.5 \mathrm{~cm}$. from the surface for five hours. During this cooling the phosphorus activity in the growth as indicated by the Geiger counter, showed a straight line. The ice was then removed, and as the temperature rose there was a peak of activity followed by a series of regular peaks at about four-hourly intervals. It is believed that this

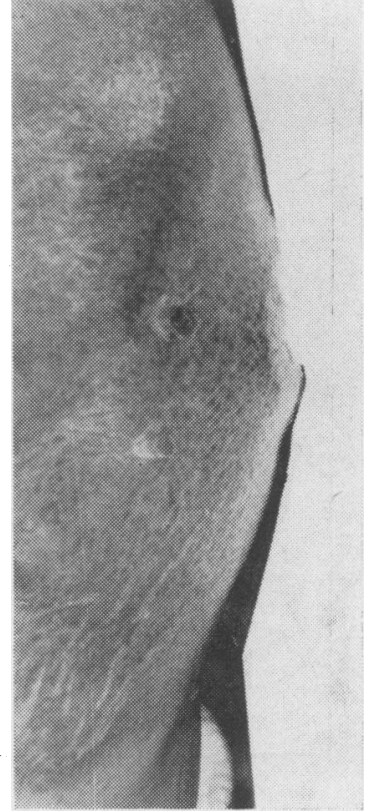

FIG. 12.-Case No. 5: After the end of the short course of D.X.R. the marks have worn off but greater regression of the edge of the malignant ulcer is seen in the upper part where treatment with D.X.R. coincided with the rise of the uptake peaks. The difference was even more marked on palpation of the mass.

behaviour has been seen by other workers using tissue cultures (Fig. 13).

The patient was given a routine course of radiotherapy to the painful mass because of the accepted radiosensitivity of this growth.

It is obvious, however, that the method of cooling the tumour is inconvenient and only easily applied to tumours of a limb, so the next step was to induce quiescence by administration of a chemotherapeutic agent. An anti-folic acid drug, methotrexate, was used as reported in the following small series of cases.

Case No. 7.-A male patient with a carcinoma of the stomach and left supraclavicular glandular metastases had uptake studies carried out on these and an irregular pattern was obtained. A course of methotrexate, $5 \mathrm{mg}$. daily, at the same time for five days was given and then stopped (Fig. 14 illustrates the result). Treatment with deep X-rays (220 kV. H.V.L. $1.5 \mathrm{~mm}$. Cu) was given to the abdominal mass (20 $\times 20 \mathrm{~cm}$. fields) and 


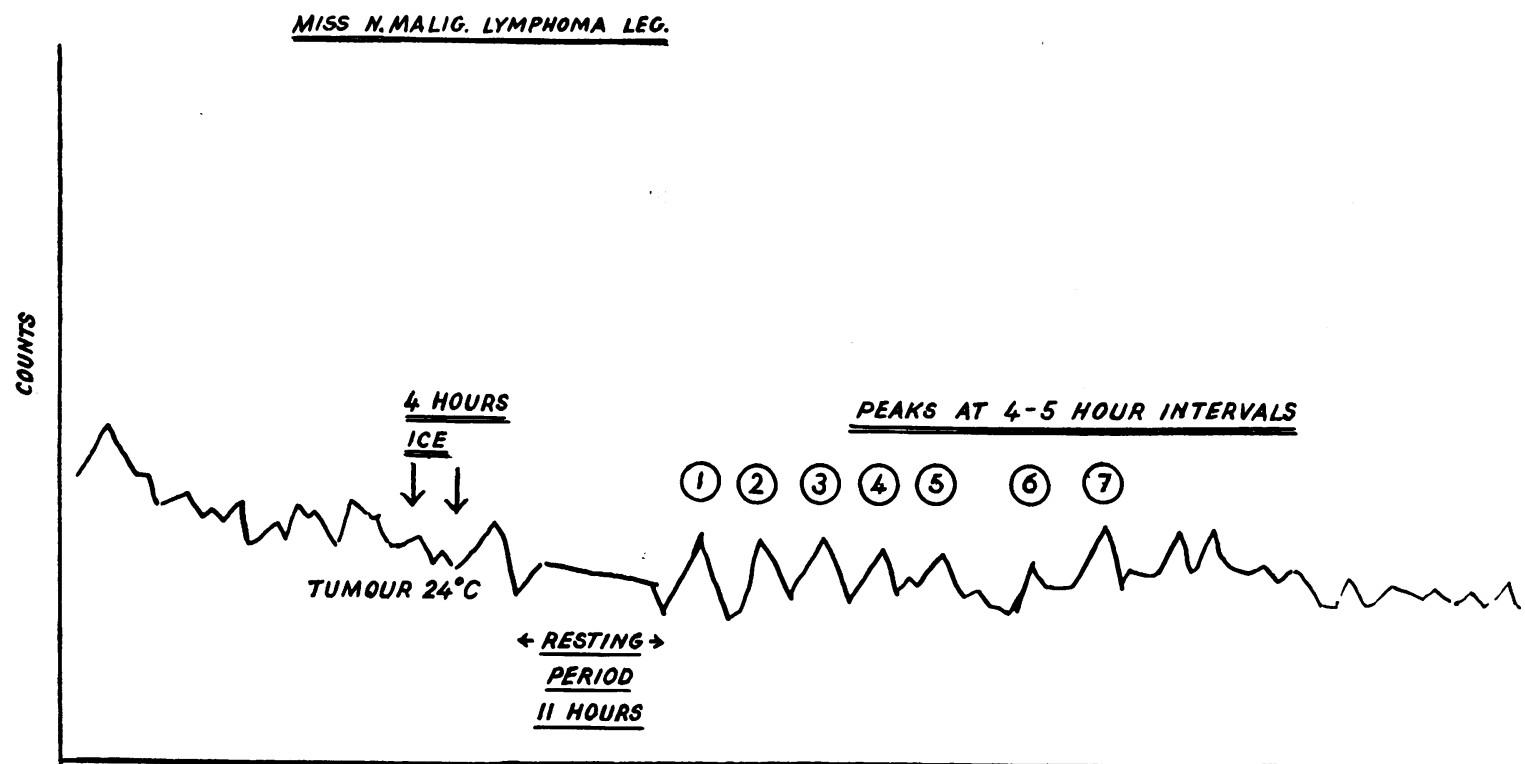

TIME IN HOURE

Fig. 13. - The tracing obtained from a malignant lymphoma, when the leg bearing it was cooled and then allowed to warm up. Note the rest period followed by regular uptake peaks.

MP. CA. STOMACH

ABD. MASB NEEDED $20 \times 20$ FIELD

(8)

(L) SUPRACLAVICULAR NODES

(6)

(5)

IRREGULAR 2 DAYS

PRIOR TO GRADH

(1)

(2) (3)

$M N$ Mhn<smiles>CCCCCCCCCCCCCCCCCC</smiles>

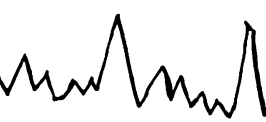

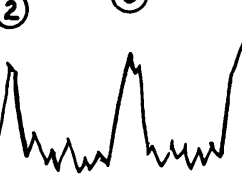

(4)
$\uparrow$<smiles>CCCC</smiles><smiles>C1CC1</smiles>

$\uparrow$

$\uparrow$

(7)

융. 


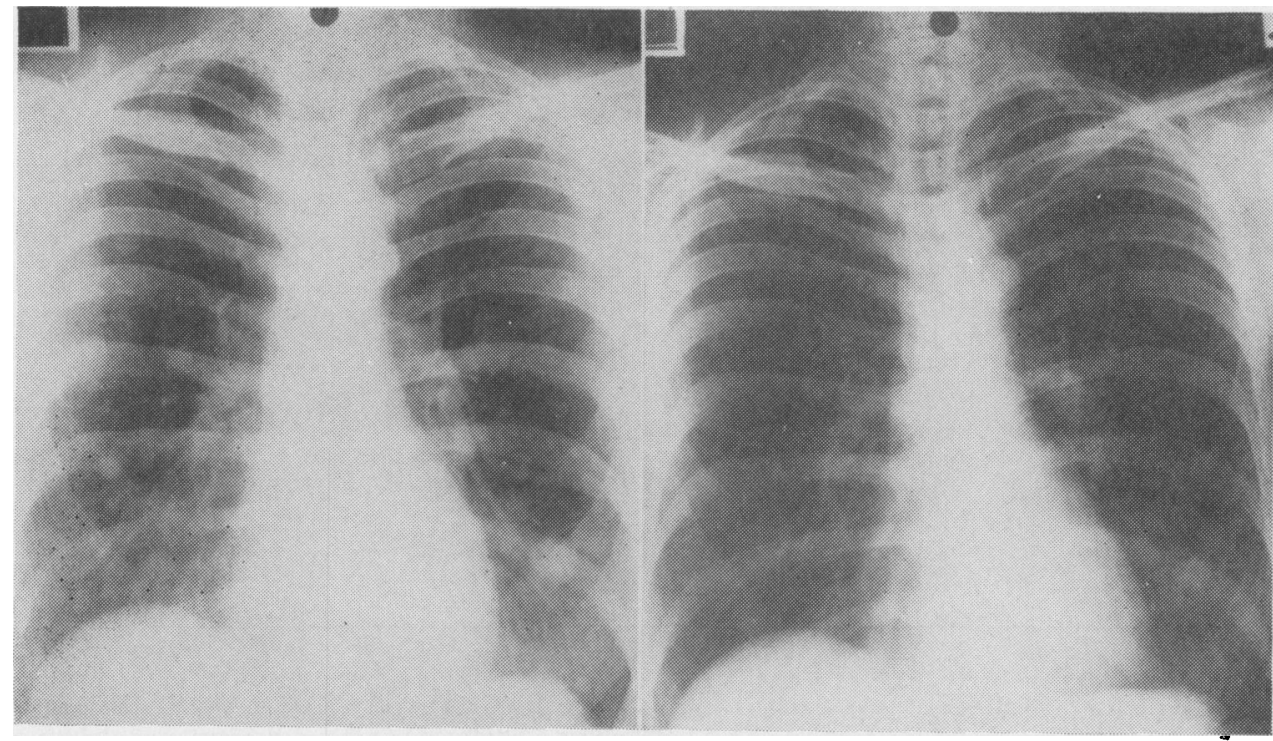

Fig. 15.-Case No. 8: The reduction in pulmonary metastases from a carcinoma of the pancreas (normally a resistant tumour) when treated with D.X.R. at the time of uptake peaks.

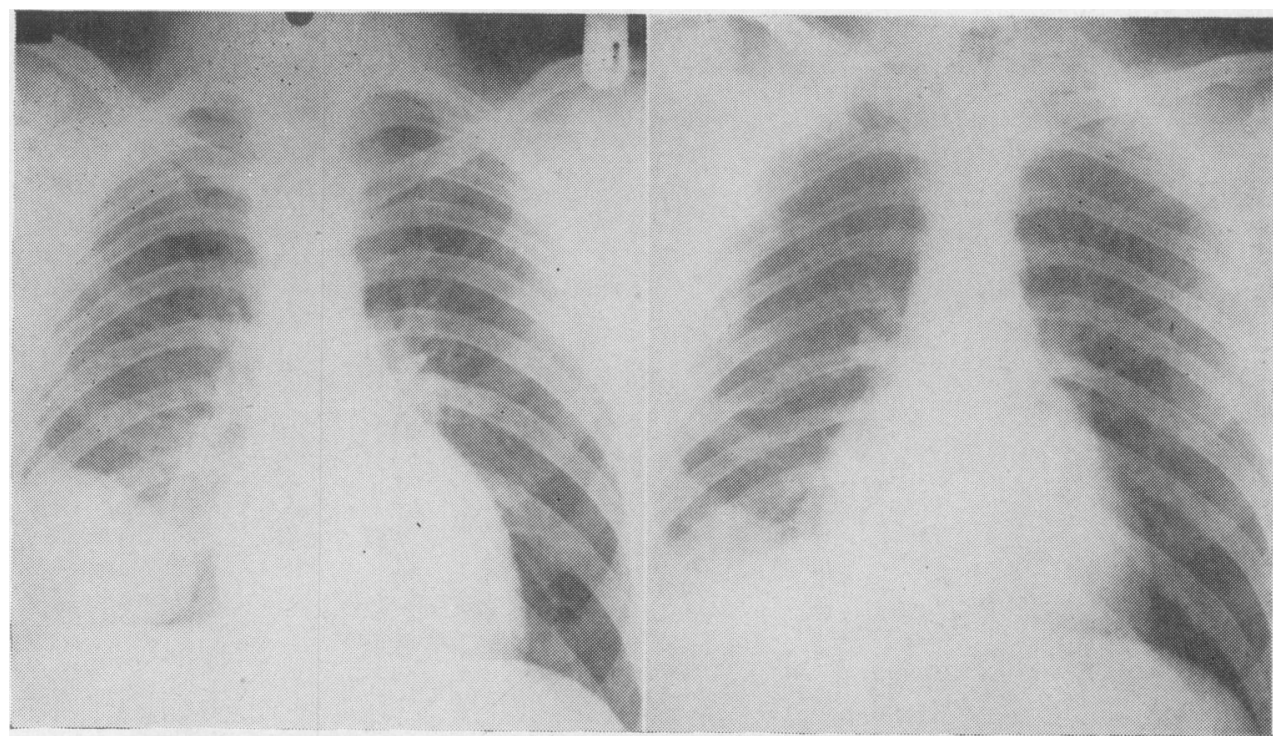

Fig. 16.-Case No. 9: The reduction in the pulmonary metastasis, from a fibrosarcoma which had been previously resistant to D.X.R., when it was treated with radiation at the times of uptake peaks.

the supraclavicular nodes. A tumour dose of 200 rads was delivered each day at the times of peak activity. By 2,000 rads the epigastric mass had considerably diminished in size and field sizes were reduced. By 3,000 rads it could no longer be felt. The supraclavicular nodes took longer to regress but had disappeared by 4,000 rads. The patient survived some seven months and then died from widespread metastases. The masses did not, however, recur in the treated areas.

Case No. 8. - This lady, aged only 44, had a fixed epigastric mass which had been proved by laparotomy and biopsy to be carcinoma of the pancreas. At a second operation a Geiger probe was buried in the mass which by then was $20 \times 12 \mathrm{~cm}$. An irregular activity pattern was shown. Methotrexate, $5 \mathrm{mg}$. daily for six days, was given and was followed by regular peaks of ${ }^{32} \mathrm{P}$ uptake occurring every 24 hours. Deep $\mathrm{X}$-ray therapy $(220 \mathrm{kV}$. as before) was then started, the treatments being timed to coincide with commencement of peaks. After 1,400 rads the tumour was much smaller, and encouraged by this she was transferred to cobalt therapy and a total tumour dose of 6,000 rads 
$M \sim$ A.M.

FIBROSARCOMA ARM

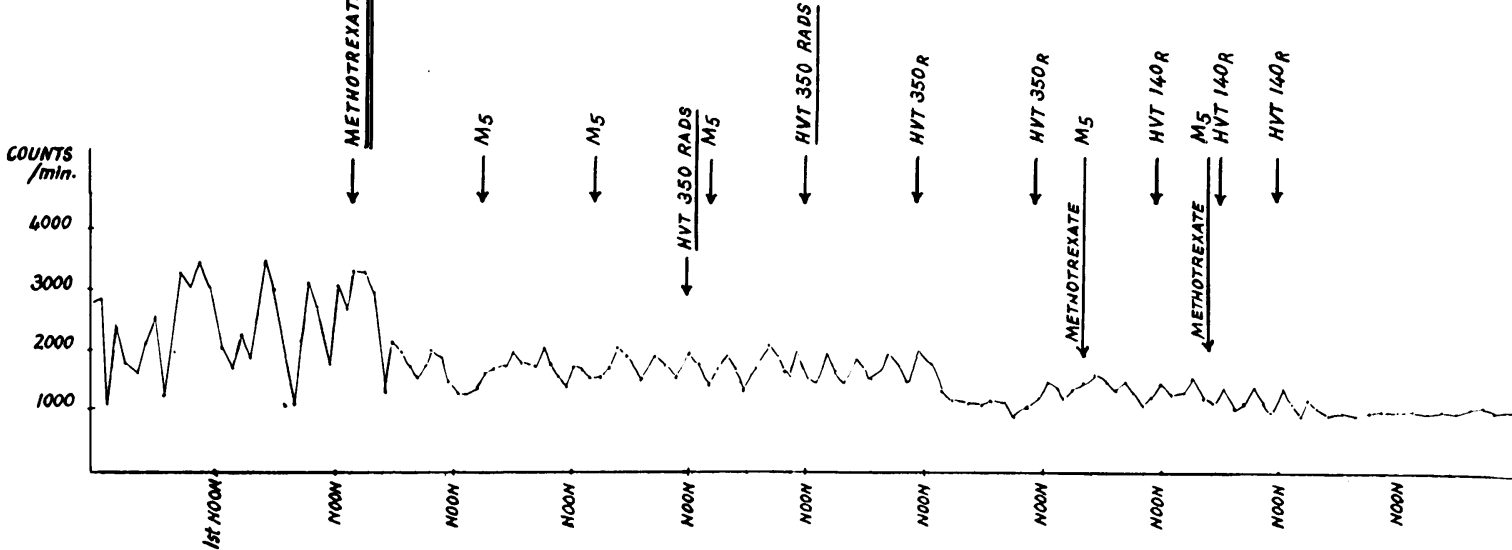

FIG. 17.-Case No. I0: This shows the pattern of ${ }^{32} \mathrm{P}$ uptake peaks obtained when methotrexate was given to a case of fibrosarcoma of forearm. D.X.R. was given at the times marked in the figure and the tumour disappeared.

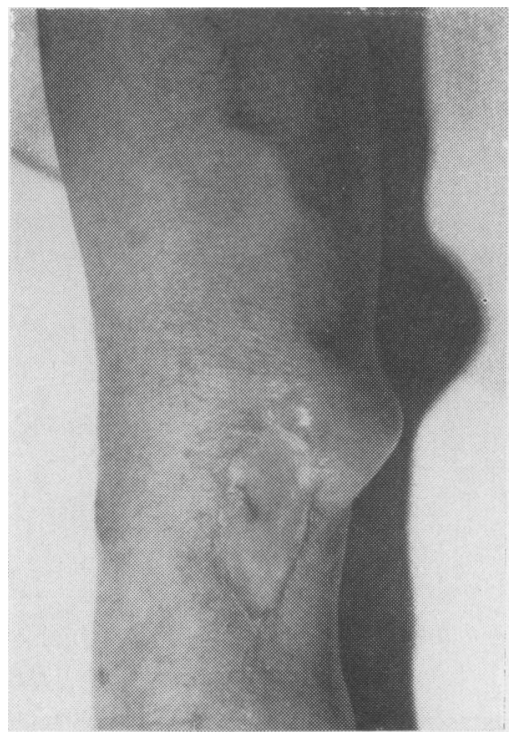

FIG. I8.-Case No. 10: Fibrosarcoma of forearm.

given over 47 days. 'The probe was only retained for I6 days, but peaks occurred consistently at the same time and treatments were given in accordance with this.

Two months after completing therapy the epigastric mass was a small, fixed remnant measuring about $5 \mathrm{~cm}$. in diameter. This has since remained unchanged. More recently, she was admitted with an enlarged node in the left supraclavicular fossa and was found to have developed multiple pulmonary metastases. The same cyclical pattern of uptake was induced with metho-

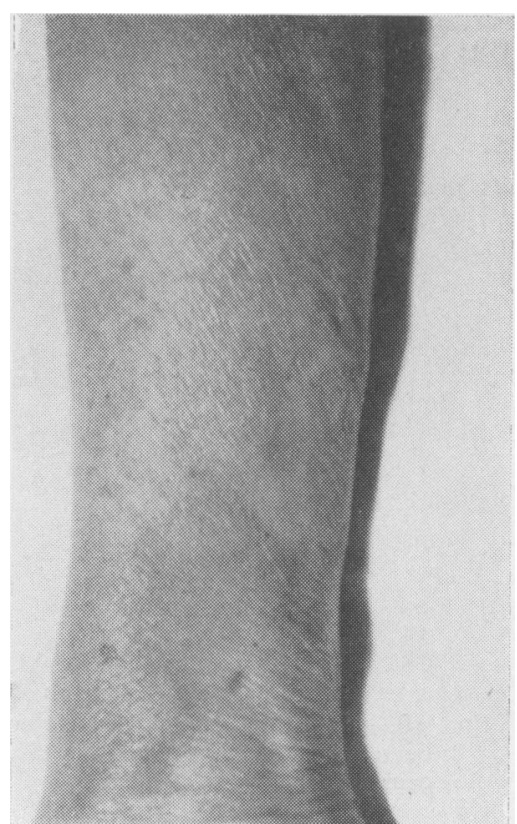

FIG. 19.-Case No. 10: Response to ${ }^{60} \mathrm{Co}$ therapy given at the times of uptake peaks.

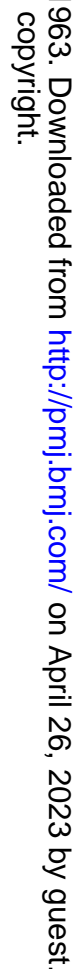

trexate, and the supraclavicular mass was used to take readings, this time with a surface counter.

The patient was treated with deep X-rays by a thoracic bath technique. A daily dose of 170 rads was given, at the times when activity appeared to increase, 
to a total dose of 2,500 rads in the centre of the chest. After only a week of treatment the X-ray appearances showed marked improvement, and the latest films show nearly complete clearance of pulmonary metastases (Fig. I 5). The supraclavicular node was just a small remnant when therapy was completed. She remained well for six months.

We have treated two cases of connective tissue sarcoma.

Case No. 9.- - Is that of a young man, aged 21 years, with recurrent pleomorphic sarcoma of fascial origin in the thigh, and a pulmonary secondary. The chest secondary had previously been given 3,500 rads of deep $\mathrm{X}$-rays at another centre with no effect. A regular pattern of peaks at 24-hour intervals was induced in the thigh tumour by means of methotrexate. Deep X-ray therapy was then given at the time of peaks to both thigh and chest. At a dose of $\mathrm{r}, 000$ rads the thigh tumour was smaller and after 3,000 rads it had completely resolved. The lung deposit was given 2,000 rads and steadily diminished after treatment, as can be seen in the X-rays (Fig. I6). In this case note especially the response to treatment at activity peaks though previously 3,000 rads given in the ordinary way did little good.

Case No. 10.- The other fibrosarcoma occurred in the forearm of an older patient and had been twice locally removed with subsequent recurrence. It was treated in the same way though the radiotherapy was from a cobalt 60 beam unit and given to a higher dose. The tumour disappeared and she now has no swelling to feel. (Fig. I 7 shows the uptake peaks obtained in her case; Figs. I 8 and 19 show the response.)

Space does not allow full description of all cases so treated up till now, but two other cases of carcinoma of the stomach are worth brief mention. Both had recurrent abdominal masses after gastrectomy. In both Geiger tubes were buried in the tumours at a second operation, and a cyclical pattern of ${ }^{32} \mathrm{P}$ uptake induced with methotrexate. Deep X-ray therapy was given and in one the tumours had become impalpable while in the other a residual mass could just be felt. Both patients felt well. It must be emphasized, of course, that this work is very recent. The therapy using methotrexate to induce a cyclical uptake and timed doses of radiation has only been used from April I962, therefore we still have to see how long-lasting the local results will be while all such patients are, of course, very likely to have more distant metastases. It will be seen that we have confined our cases to types of tumour which are generally radioresistant so that a good result from the method might have some significance.

\section{Discussion}

We have yet to treat a control series with radiotherapy given away from the peak times to demonstrate whether or not we are only seeing a synergistic effect of the radiotherapy and methotrexate. The case which was treated during a natural cycle (Case No. 5) is a help here. We also wish to study the degree to which vascular changes occur at the times of the 'peaks'.

For detailed research, as we have already stated, we must emphasize that continuous recording of ${ }^{32} \mathrm{P}$ uptake appears essential. However, for a few simple procedures, e.g. assessment of hormone-dependence by measuring uptake before and after administration of hormones, or assessing response to therapy, it may sometimes be enough to take single, reasonably prolonged, uptake readings before and after administration of the drug. In this case, the ${ }^{32} \mathrm{P}$ dose will also be divided in two parts.

The work so far described appears to be leading to at least an increased success in the treatment of malignant growths which are both inoperable, and resistant to conventional methods of radiotherapy and chemotherapy. It has disclosed a number of possibilities which demand investigation. Some of them are of the most immediate and practical interest. For example, the cases in which growth activity is suppressed by means of an anti-metabolite drug and then released, produce peaks of activity which demand radiotherapy at times which are very difficult to fit into the work of a department.

Treatment must be available 'round the clock', day and night. We should if possible arrange the dosage and timing of the controlling drug so that activity peaks occur at chosen times, to allow a timetable of work to be constructed. It would not, of course, follow that such controlled peaks would occur daily; they might occur on alternate days or at longer intervals. We intend to go into this problem as soon as possible.

Several alternative schemes of therapy suggest themselves. For example:

(a) When the first peak occurs a further large dose of the same antimetabolic drug can be given, as a means of therapy, then a further dose at the next peak and so on. However, this may be expected to result in long periods of inhibition, which may prolong treatment beyond the time for which a counter can remain in position.

(b) We may treat with radiation at such peaks. In our experience this has the advantage that radiation does not immediately inhibit further peaks. This may be due to the fact that, in a normally radioresistant tumour, cells which were resting at the earlier peak have been unaffected by the radiation. Alternatively, in the case of radiation there may be a delay period between radiation and the appearance of the reaction.

(c) Treatment at the peaks may be with alkylating agents (or other types of chemotherapy) 
which are to a varying degree radiomimetic and may represent a compromise between $(a)$ and $(b)$ above. Certainly radiomimetic drugs will be easier to give than radiation in the middle of the night!

Choice between chemotherapy and radiotherapy at activity peaks may well depend on whether a tumour is generalized or relatively localized. When chemotherapy is used rapid administration of a drug which acts immediately (e.g. nitrogen mustard) would appear to be called for rather than slow infusion, whether local or systemic.

The enhanced response of radioresistant tumours when treated at activity peaks, may give us more information about the nature of radioresistance and radiosensitivity. It is difficult to imagine that when a radiosensitive tumour is treated daily at the same hour and duly disappears, all the cells have been caught at a time of activity; surely some of them were inactive every time therapy was given, and in any case what about the tumours (e.g. small rodent ulcers) which are cured only after a few doses or a single X-ray treatment? It is difficult to escape the conclusion that radiosensitive tumours are so because the cells are sensitive when they are resting. Conversely, we have reported in this paper cases of normally radioresistant tumours which were sensitive provided they were treated at peaks of activity. We might deduce, therefore, that resistant tumours include those whose cells are insensitive to radiation while in a resting state.

We have noticed in these resistant cases that the tumours become resistant as soon as a peak has passed, but they may be sensitive if radiotherapy has been given shortly before a peak. Relatively sensitive growths may include those in which the cells are sensitive for a considerable time before the active stage is detectable with ${ }^{32} \mathrm{P}$.

It has been suggested to us that anaplastic growths may tend to show a more rapid cycle of peaks. This remains to be investigated, but the suggestion is consistent with the generally greater radiosensitivity of anaplastic growths, since the more frequent the peaks are the less proportion of time cells will be in the unresponsive stage immediately following activity.

We have remarked above that radiotherapy does not appear to suppress activity quickly, but in due course it does and in the case of the more resistant tumours in which, if the speculations above have any truth, the cells are resistant when resting, it would appear logical to suspend treatment until activity has commenced again. Possibly, therefore, even if radiotherapy is given alone with no controlling drug it should sometimes be phased, and a succession of short courses of treatment given. Here we have yet another@ matter which should be investigated by phos- $\bar{z}$ phorus uptake. Indeed the points which need $\stackrel{\mathbb{\Phi}}{\varrho}$ investigation appear to be legion.

We are beginning to examine the use of a $\overrightarrow{\vec{F}}$ colchicine derivative as a controlling drug instead $\stackrel{?}{?}$ of methotrexate, and viniblastine sulphate is? being tried. We do not yet know the ideal timing $\frac{\bar{\sigma}}{\bar{c}}$ or even dosage of either the administration of the $\frac{\text { क }}{\widehat{D}}$ controlling drug or the radiotherapy or chemo- $\varnothing$ therapy to follow, and this in any case may be expected to vary from one type of tumour to $\overrightarrow{0}$ another. We must discover at just what points in the cycle of activity tumours are most sensitive $\vec{\sigma}$ to treatment.

The records will also give information on the $\frac{\mathbb{C}}{3}$ duration of action of the various drugs. The $\frac{3}{\omega}$. possession of the continuous record of phosphorus uptake may be likened to directing a campaign of under continuous visual control, even if rather $\vec{\sim}$ imperfect, compared with blind shooting at of invisible targets.

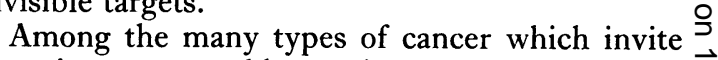
attention, we would mention one resistant and $\vec{z}$ relatively rare tumour. That is osteogenic sarcoma. So frequently the patient already has secondaries $\vec{\theta}$ in the lungs, visible or invisible, when he $\&$ first seen, and failure of cure by amputation usually due to this cause.

A trial of this technique may be made in cases of osteogenic sarcomata of limbs, which already have chest secondaries when first seen. In such $\frac{0}{D}$ cases a ${ }^{32} \mathrm{P}$ probe may be inserted into the primary $\stackrel{\nwarrow}{\varrho}$ tumour. This involves no important risk when $\overrightarrow{\overrightarrow{0}}$ dissemination of the growth has already occurred. If we are fortunate the primary will develop cycles of activity (as we have already seen in cases of fibrosarcoma) and then the chest secondaries should be irradiated at the time of the cycles (cf. our cases with disappearance of chest secondaries). If the chest secondaries disappear then the primary may be amputated or irradiated.

Although such investigations of special interest on the rarer tumours may be very valuable, we must not forget that most effort should be concentrated on the commoner types of case which will more quickly allow of statistical results. We have $N$ so far concentrated our effort on clinical research $N$ and the development of the apparatus, because those are the things which we have been equipped to do. There is in any case no doubt that it is essential to develop the apparatus for continuous recording so that it is easy to use and gives all useful information in a way which is reliable and easy to read.

A complete biochemical research into the implications of this work would provide many of the answers required for the most effective

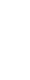


treatment, but that would be at very large undertaking which the present group of workers could not attempt. However, we must consider the possibility of a simple investigation on a histological basis, involving the counting of cells in different phases of activity and the correlation of this with the ${ }^{32} \mathrm{P}$ uptake tracings. In addition, we propose to measure temperature variations and changes in blood flow within the tumour, in order to relate these to the activity cycles or even natural diurnal variations. We must again stress the fact that only careful and complete observations will produce results.

The other contributors to this paper must express their indebtedness to one of their number, without whose devotion the work would never have been accomplished. He has carried out nearly all the practical clinical work, and has been ready at every hour of the day and night to treat our patients whenever an activity peak occurred. It was this readiness which, in the breast case (No. 5), uncovered the potentialities of radiotherapy given at activity peaks.

Finally, it must be stressed that this is a report of research in progress and the work must in no way be regarded as complete. Many 'teething problems' have been overcome and we shall soon have sufficient apparatus to permit many more cases to be studied, and to see if the encouraging early results truly indicate the trend to come.

Figs. 4, 5, 6 and 8 are reproduced by kind permission of the Editor of the Lancet.

\section{REFERENCES}

Burk, D., Laszlo, J., Hunter, J., Wight, K., and Woods, M. (i960): The Differential Metabolic Responses of Susceptible and Resistant Mouse Leukæmia Cells to 8-azaguanine, $\mathcal{F}$. nat. Cancer Inst., $24,57$.

Hale, Brendan T. (I96I): A Technique for Studying Human Tumour Growth in Vivo, Lancet, ii, 345.

Howard, Alma, and Pelc, S. R. (195I): Synthesis of DNA and Nuclear Incorporation of $\mathrm{S}^{35}$ as Shown by Autoradiographs. Ciba Foundation Conference on Isotopes in Biochemistry. London: J. \& A. Churchill.

- (1956): Influence of Radiation on DNA Metabolism. Ciba Foundation Symposium on Ionising Radiations and Cell Metabolism. London: J. \& A. Churchill.

Kellfrshohn, C., Herzberg, B., and Martin, J. (1958): Possibilité et Interêt de la Detection Externe par 'Bremsstrahlung' de Radiophosphore $\mathrm{P}^{32}$ dans l Organisme', Strahlentherapie, 38, $33 \mathrm{I}$.

Mril, H. G. (1958): Kinetics and Distribution of $\mathrm{P}^{32}$ as Measured by Bremsstrahlung in Diagnosis and Therapy . Second U.N. International Conference on the Peaceful Uses of Atomic Energy, Geneva, A/Conf. I 5/P/978, 26, 224. (1959): The Distribution of a Pure Beta-Emitter in the Human Body. Problems and Preliminary Results of Bremsstrahlung Measurements in Vivo. Medical Radio-isotope Scanning, International Atomic Energy Agency, Vienna, pp. 125/142. 